\title{
Synthesis of a spatial 3-RPS parallel manipulator based on physical constraints
}

\author{
NALLURI MOHAN RAO \\ Department of Mechanical Engineering, University College of Engineering, \\ Jawaharlal Nehru Technological (JNT) University Kakinada, Kakinada 533003 \\ e-mail: nmohan72@rediffmail.com
}

MS received 5 August 2009; revised 1 April 2010; accepted 23 August 2010

\begin{abstract}
The range of motion of the moving platform of a spatial 3-RPS parallel manipulator will be greatly influenced by the physical constraints such as limits on the lengths of the limbs and the range of motion of the spherical joints. Therefore, while synthesizing the parallel manipulator, the physical constraints have to be considered. Synthesis of the manipulator involves determination of the architectural parameters of the manipulator so that a point on the moving platform passes through a prescribed set of positions in space. This paper presents a synthesis procedure that determines location and direction of revolute joints and location of spherical joints along with orientation of sockets of spherical joints, considering the physical constraints. The synthesis procedure is demonstrated through a numerical example.
\end{abstract}

Keywords. 3-RPS parallel manipulator; physical constraints; synthesis.

\section{Introduction}

The need for the large load carrying capacity, high accuracy in positioning the end effector and greater accelerations in robot applications lead to the development of different types of parallel manipulators. The parallel manipulators with limited (less than six) degrees of freedom (DOF) gained prominence over those (Gough \& Whitehall 1962; Stewart 1965) with 6 -DOF because of their complicated analysis and more over several applications require less than 6-DOF only.

A Spatial parallel manipulator with three revolute $(\mathrm{R})$-prismatic $(\mathrm{P})$-spherical and $(\mathrm{S})$ chains has 3-DOF (Lee \& Shah 1987, 1988). Therefore, its end effector cannot be positioned in space at will (Tsai \& Kim 2003). Hence the manipulator has to be synthesized in order that its end effector passes through a set of prescribed positions in space.

The range of motion of the moving platform or the workspace of a spatial 3-RPS parallel manipulator will be greatly influenced by the physical constraints (Lee \& Shah 1987) such as the limits on the lengths of the limbs and the range of motion of the spherical joints. Therefore, the physical constraints are to be considered while synthesizing the parallel manipulator. This work presents kinematic synthesis of a spatial 3-RPS parallel manipulator in order that a point on the moving platform passes through a set of prescribed positions in space. A procedure for 
the synthesis of a spatial 3-RPS parallel manipulator was presented in (Rao \& Rao 2009) where the constraints on the range of motion of spherical joints were considered for determining the location and direction of revolute joints and location of spherical joints for a fixed value of orientation of sockets of spherical joints. But the synthesis procedure presented in this work determines orientation of sockets of spherical joints also. It was shown in (Lee \& Shah 1987) that the orientation of sockets of spherical joints has an influence on the workspace of the manipulator and hence this parameter is considered as one of the design parameters of the synthesis procedure.

Chen \& Roth (1967) presented the synthesis of a RPS serial chain for seven positions. Su \& McCarthy (2003) have carried out the synthesis of the RPS chain for a number of positions up to ten. They provided analytical solutions to the problem for the six through eight position cases and solved the problem numerically using the polynomial continuation method for the nine and ten position cases. A procedure for the synthesis of a spatial 3-RPS parallel manipulator for any number of positions using the Least Square Technique was presented in (Rao \& Rao 2006).

\section{Geometry and design equations}

The Spatial 3-RPS parallel manipulator consists of a moving platform $\{\mathrm{M}\}$ connected to the fixed base $\{\mathrm{F}\}$ by three identical limbs as shown in figure 1 . Each limb consists of an upper and a lower member connected by means of a prismatic joint $\mathbf{P}$. The prismatic joint is to be driven by a linear actuator. A revolute joint $\boldsymbol{R}$ at $A_{i}(i=1,2,3)$ connects the lower member of each limb to the fixed base and a spherical joint $S$ at $B_{i}$ connects the upper member to the moving platform. The axes of the revolute joints are $J_{1}, J_{2}$ and $J_{3} . \mathbf{a}_{i}=\left[\begin{array}{lll}a_{x} & a_{y} & a_{z}\end{array}\right]^{T}$ is the position vector of the revolute joint $A_{i}(i=1,2,3)$ with respect to the fixed frame $\mathbf{X Y Z}$ and $\mathbf{b}_{i}=\left[\begin{array}{lll}b_{x} & b_{y} & b_{z}\end{array}\right]^{T}$ is the position vector of the spherical joint $B_{i}$ with respect to the moving frame xyz. The direction of each revolute joint with respect to the fixed frame is indicated by a unit vector along its axis $\mathbf{j}_{i}=\left[\begin{array}{lll}j_{x} & j_{y} & j_{z}\end{array}\right]^{T}$.

There are, on the whole, eight links and nine (three revolute, three prismatic and three spherical) joints. The degrees of freedom for a spatial mechanism can be calculated using the Grubler-Kutzbach criterion,

$$
F=\lambda(n-j-1)+\sum_{i} f_{i}
$$

where $F$ is degrees of freedom of a mechanism, $f_{i}$ is degrees of relative motion permitted by joint $i, j$ is number of joints in a mechanism, assuming that all joints are binary, $n$ is number of links in a mechanism, $\lambda$ is degrees of freedom of a space in which a mechanism is intended to function.

The manipulator has three degrees of freedom, two rotational and one translatory (Lee \& Shah 1988).

Let $R_{i}$ and $\mathbf{p}_{i}$ denote the rotation matrix and the position vector of a reference point on the moving platform respectively at the $i^{\text {th }}$ specified pose with respect to the Fixed frame XYZ. A constraint equation (Tsai \& Kim 2003) based on the assumption that the prismatic joint axis is perpendicular to the revolute joint axis can be written as,

$$
\overline{A B} \cdot \mathbf{j}=0
$$




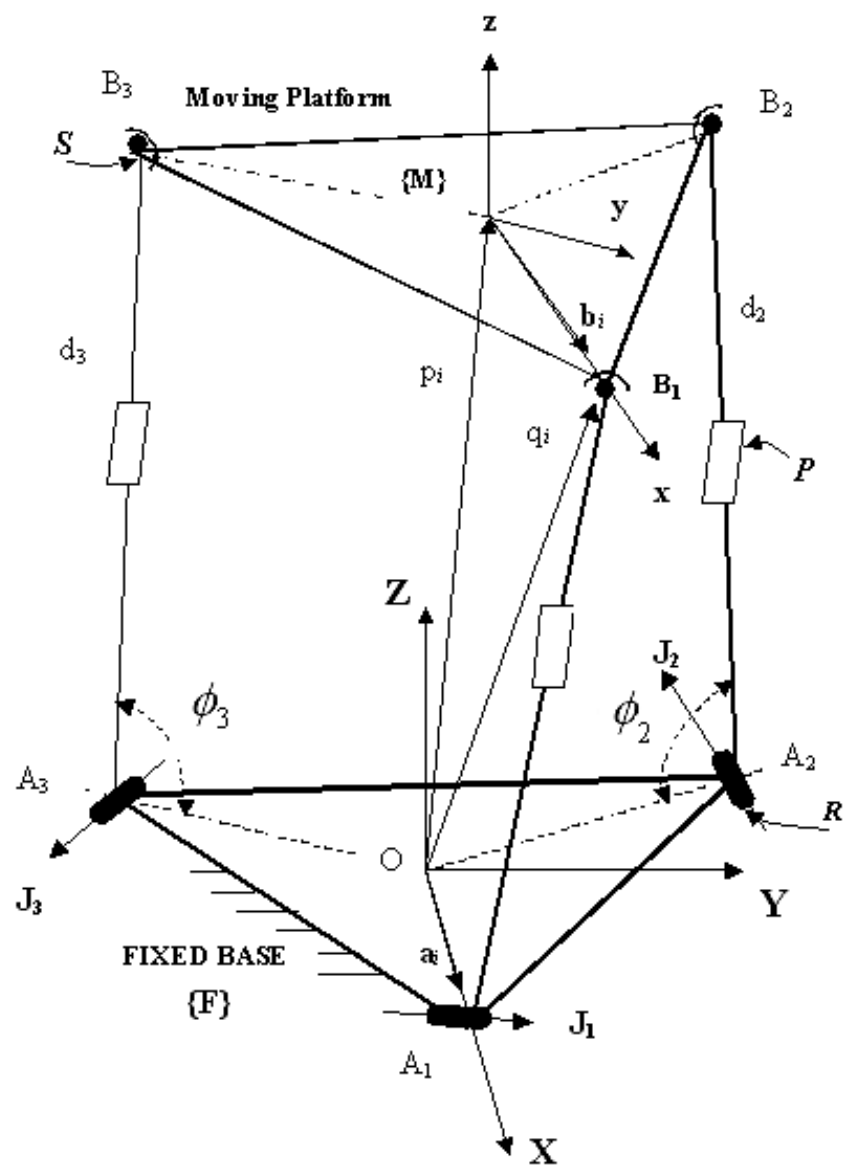

Figure 1. Spatial 3-RPS parallel manipulator.

or

$$
\left[R_{i} \mathbf{b}+\mathbf{p}_{i}-\mathbf{a}\right]^{T} \mathbf{j}=\left[\mathbf{q}_{i}-\mathbf{a}\right]^{T} \mathbf{j}=0 \quad \text { for } \quad i=1,2,3, \ldots, n,
$$

where $\mathbf{q}_{i}$ is the position vector of the spherical joint with respect to the fixed frame. The design variable a can be eliminated by subtracting Eq. (2) for $i=1$ from Eq. (2) for $i=2$ to $n$. This results in

$$
\left[R_{i}^{\prime} \mathbf{b}+\mathbf{p}_{i}^{\prime}\right]^{T} \mathbf{j}=0 \quad \text { for } \quad i=2,3, \ldots, n,
$$

where

$$
R_{i}^{\prime}=R_{i}-R_{1} \text { and } \mathbf{p}_{i}^{\prime}=\mathbf{p}_{i}-\mathbf{p}_{1}
$$

\section{Physical constraints}

The physical constraint considered here is the limit on the range of motion of the spherical joint due to the socket and it may be expressed as the maximum angle between the axis of symmetry of the spherical joint and the link, $\psi_{\max }$ (Lee \& Shah 1987). The maximum angle 


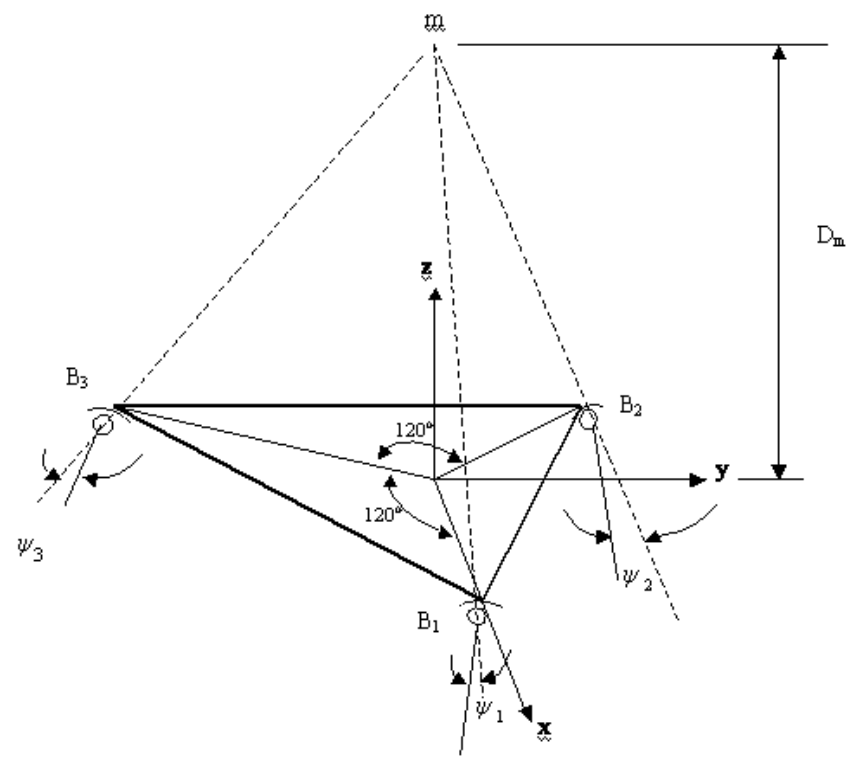

Figure 2. Arrangement of the spherical joint on the moving platform.

$\psi_{\max }$ of the spherical joint has significant influence on the orientation of moving platform. Hence the constraint is considered while determining the location of the revolute joint for a given set of $\mathbf{b}_{1}, \mathbf{b}_{2}, \mathbf{b}_{3}, \mathbf{j}_{1}, \mathbf{j}_{2}$ and $\mathbf{j}_{3}$ vectors. The angle between the axis of symmetry of the spherical joint and the link $\psi_{i}$ can be expressed as a function of the position and orientation of the moving platform (Lee \& Shah 1987).

Figure 2 shows the location of the spherical joint with respect to moving platform. Let $N$ be the normal vector of a plane containing the spherical joints and is given by,

$$
N=a_{1} I+b_{1} J+c_{1} K
$$

where $I, J$ and $K$ are the unit vectors along $X, Y$ and $Z$ axes of the co-ordinate system fixed to the base platform.

The equation of the corresponding plane in normal form is,

$$
A x+B y+C z=d,
$$

where $A, B$ and $C$ are the components of the unit normal vector.

$$
\text { Also, } \quad N=\overline{B_{1} B_{2}} \times \overline{B_{2} B_{3}} \text {. }
$$

$\overline{B_{1} B_{2}}$ is a vector directed from the spherical joint $B_{1}$ to $B_{2}$ and $\overline{B_{2} B_{3}}$ is a vector directed from the spherical joint $B_{2}$ to $B_{3}$. On comparing the equations Eq. (4) and Eq. (6), the components of the normal vector $N$ can be determined. The Cartesian co-ordinates of each of the spherical joints with respect to the base frame XYZ can be expressed as,

$$
\left[\begin{array}{l}
B_{i} \\
1
\end{array}\right]=[T]\left[\begin{array}{c}
\mathbf{b}_{i} \\
1
\end{array}\right],
$$


where $B_{i}$ and $\mathbf{b}_{i}$ are the position vectors of the spherical joint with respect to the base frame $\mathbf{X Y Z}$ and the moving frame xyz respectively and $[T]$ is a homogeneous transformation matrix given by Tsai (1999),

$$
[T]=\left[\begin{array}{cccc}
R_{11} & R_{12} & R_{13} & x_{c} \\
R_{21} & R_{22} & R_{23} & y_{c} \\
R_{31} & R_{32} & R_{33} & z_{c} \\
0 & 0 & 0 & 1
\end{array}\right],
$$

where $x_{c}, y_{c}$ and $z_{c}$ are co-ordinates of the origin of the frame $\mathbf{x y z}$ attached to the moving platform.

The axis of symmetry of each socket of the spherical joints intersects the normal of the plane at point ' $m$ '. The equation of the line along the normal and passing through the point $\left(x_{c}, y_{c}, z_{c}\right)$ is,

$$
\frac{x-x_{c}}{a_{1}}=\frac{y-y_{c}}{b_{1}}=\frac{z-z_{c}}{c_{1}} .
$$

But,

$$
A=\frac{a_{1}}{\sqrt{a_{1}^{2}+b_{1}^{2}+c_{1}^{2}}} ; \quad B=\frac{b_{1}}{\sqrt{a_{1}^{2}+b_{1}^{2}+c_{1}^{2}}} \quad \text { and } \quad C=\frac{c_{1}}{\sqrt{a_{1}^{2}+b_{1}^{2}+c_{1}^{2}}}
$$

Therefore Eq. (9) can also be written in terms of the co-ordinates $\left(x_{m}, y_{m}, z_{m}\right)$ of the point $m$, which is the point of intersection of the axis of symmetry of each socket with the normal of the moving platform, as

$$
\frac{x_{m}-x_{c}}{A}=\frac{y_{m}-y_{c}}{B}=\frac{z_{m}-z_{c}}{C}=D_{m} .
$$

Hence, the co-ordinates of the point ' $m$ ' can be obtained as,

$$
x_{m}=x_{c}+A \cdot D_{m} ; \quad y_{m}=y_{c}+B \cdot D_{m} \quad \text { and } \quad z_{m}=z_{c}+C \cdot D_{m} .
$$

Similarly, the equation of the line passing through the $i^{\text {th }}$ spherical joint and the $i^{\text {th }}$ pin joint is,

$$
\frac{x-a_{i x}}{x_{B i}-a_{i x}}=\frac{y-a_{i y}}{y_{B i}-a_{i y}}=\frac{z-a_{i z}}{z_{B i}-a_{i z}} \quad(i=1,2,3) .
$$

Again, the equation of the line passing the point ' $m$ ' and the $i^{\text {th }}$ spherical joint is,

$$
\frac{x-x_{B i}}{x_{m}-x_{B i}}=\frac{y-y_{B i}}{y_{m}-y_{B i}}=\frac{z-z_{B i}}{z_{m}-z_{B i}} \quad(i=1,2,3) .
$$

Therefore, the angle $\psi_{i}$ between the two lines described by the Eq. (13) and Eq. (14) is given by the equation Eq. (15) for $i=1,2,3$ as shown below,

$$
\begin{aligned}
\cos \psi_{i}= & \frac{1}{\sqrt{\left(x_{B i}-a_{i x}\right)^{2}+\left(y_{B i}-a_{i y}\right)^{2}+\left(z_{B i}-a_{i z}\right)^{2}}} \\
& * \frac{\left(x_{B i}-a_{i x}\right)\left(x_{m}-x_{B i}\right)+\left(y_{B i}-a_{i y}\right)\left(y_{m}-y_{B i}\right)+\left(z_{B i}-a_{i z}\right)\left(z_{m}-z_{B i}\right)}{\sqrt{\left(x_{m}-x_{B i}\right)^{2}+\left(y_{m}-y_{B i}\right)^{2}+\left(z_{m}-z_{B i}\right)^{2}}},
\end{aligned}
$$

where $0<\psi_{i}<\psi_{\max } / 2$. 
Since, in the Eq. (15), the parameter $D_{m}$ exists implicitly in terms of $x_{m}, y_{m}$ and $z_{m}$, it will also be one of the influencing parameters of range of motion of the moving platform of the manipulator. The affect of the parameter, $D_{m}$ on the size and shape of workspace of the manipulator is also shown in $\mathrm{X}-\mathrm{Z}$ and $\mathrm{Y}-\mathrm{Z}$ plots of its work envelope presented in (Lee \& Shah 1987). Hence, this parameter is to be considered while synthesizing the manipulator in order that a point on the moving platform passes through a number of prescribed positions in space, satisfying the physical constraints on the motion of spherical joints.

\section{Synthesis procedure}

The synthesis procedure consists of the determination of the vectors $\mathbf{a}_{i}, \mathbf{b}_{i}, \mathbf{j}_{i}$ and a parameter that represents the orientation of the spherical joint in order that a reference point on the moving platform passes through a set of prescribed positions in space. Each position is defined by the position vector $\mathbf{p}_{i}(i=1,2, \ldots, n)$ and rotation matrix $R_{i}(i=1,2, \ldots, n)$ with respect to the fixed frame XYZ. The synthesis is carried out in two steps. The first step consists of the determination of the vectors $\mathbf{b}_{i}$ and $\mathbf{j}_{i}$ and the second step consists of the determination of the vector $\mathbf{a}_{i}$ along with the parameter $D_{m}$ that represents the orientation of the spherical joint socket with respect to the moving platform. The constraints on the range of motion of the spherical joints are considered in the second step of the synthesis.

\subsection{Determination of the location of spherical joints $\mathbf{b}_{i}$ and direction of revolute joints $\mathbf{j}_{i}$}

Determination of the location of spherical joint $\mathbf{b}$ and direction of revolute joint $\mathbf{j}$ may be carried out by solving the unconstrained optimization problem,

$$
\text { minimization of error, } e=\sum_{i=2}^{n}\left(\left[R_{i}^{\prime} \mathbf{b}+\mathbf{p}_{i}^{\prime}\right]^{T} \mathbf{j}\right)^{2} \text {. }
$$

Hence the task is to determine the optimal solution set of $b_{x}, b_{y}, b_{z}, j_{x}, j_{y}$ and $j_{z}$ for minimum value of the error $e$ of Eq. (16) in order to satisfy $\mathbf{p}_{i}$ and $R_{i}$ for $i=1,2, \ldots, n$.

\subsection{Determination of the location of revolute joints $\mathbf{a}_{i}$ and the parameter $D_{m i}$}

The location of the revolute joint $\mathbf{a}_{i}$ and the value of the parameter $D_{m}$ for a given set of $\mathbf{b}_{1}, \mathbf{b}_{2}$, $\mathbf{b}_{3}, \mathbf{j}_{1}, \mathbf{j}_{2}$ and $\mathbf{j}_{3}$ vectors may be obtained by optimizing (minimizing) the objective function,

$$
\text { min. of error, } \varepsilon=\left\{\left(a_{x}-q_{1 x}\right) j_{x}+\left(a_{y}-q_{1 y}\right) j_{y}+\left(a_{z}-q_{1 z}\right) j_{z}\right\}_{i} \quad i=1,2,3 \text {. }
$$

Subject to constraints:

$$
\psi_{i k}<\psi_{\max } / 2 \quad i=1,2,3 \text { and } k=1,2, \ldots, n,
$$

where $n$ is the number of positions and $\left[\begin{array}{lll}q_{1 x} & q_{1 y} & q_{1 z}\end{array}\right]^{T}$ is the location of the ball joint when the moving platform is at position 1 .

\section{Numerical example}

The proposed method of the synthesis of a manipulator is demonstrated by considering the ten positions and orientations of the moving platform given in the table 1 (Rao \& Rao 2009). $p_{x}, p_{y}, p_{z}$ are the positions in meters and $\theta_{x}, \theta_{y}, \theta_{z}$ are the Euler angles in degrees defined with respect to three successive rotations about the fixed $\mathbf{X}, \mathbf{Y}$ and $\mathbf{Z}$ axes. 
Table 1. Ten sets of location and orientation of the moving platform.

\begin{tabular}{lrccrrr}
\hline S. No. & $p_{x}$ (meters) & $p_{y}$ (meters) & $p_{z}$ (meters) & \multicolumn{1}{c}{$\theta_{x}(\mathrm{deg})$} & $\theta_{y}(\mathrm{deg})$ & \multicolumn{1}{c}{$\theta_{z}(\mathrm{deg})$} \\
\hline 1 & 0.000000 & 0.000000 & 2.0000 & 0.000000 & 0.000000 & 0.000000 \\
2 & -0.020746 & 0.024462 & 2.621373 & -6.224981 & 13.178057 & -0.719746 \\
3 & -0.038051 & 0.0246859 & 2.817743 & -5.098402 & 16.589436 & -0.737934 \\
4 & -0.039095 & -0.011559 & 2.917504 & 2.416787 & 16.242535 & 0.344925 \\
5 & -0.021316 & -0.033523 & 2.904463 & 7.963235 & 14.158188 & 0.990493 \\
6 & 0.028352 & -0.049108 & 2.831955 & 16.949255 & 9.554010 & 1.426765 \\
7 & 0.135818 & 0.002199 & 2.655059 & $30 \cdot 211072$ & -0.233396 & -0.062999 \\
8 & 0.118370 & 0.030952 & 2.653171 & 28.442727 & -3.504413 & -0.888402 \\
9 & 0.080189 & 0.026062 & 2.706538 & 23.437071 & -3.605556 & -0.748127 \\
10 & 0.040164 & 0.014722 & 2.787508 & 16.534539 & -2.898580 & -0.422289 \\
\hline
\end{tabular}

\subsection{Determination of the location of spherical joints $\mathbf{b}_{i}$ and direction of revolute joints $\mathbf{j}_{i}$}

Some of the results obtained by minimizing the objective function Eq. (16) using a nontraditional optimization method - Genetic Algorithms (Deb 2001) are (Rao \& Rao 2009):

$$
\begin{aligned}
{\left[\begin{array}{rlllll}
\mathbf{b}_{x} & \mathbf{b}_{y} & \mathbf{b}_{z} & \mathbf{j}_{x} & \mathbf{j}_{y} & \mathbf{j}_{z}
\end{array}\right]^{T}=} & {\left[\begin{array}{lllllll}
1.2637 & 0.2828 & -0.1989 & 0.207 & -0.9773 & 0.04483
\end{array}\right]^{T} ; } \\
& {\left[\begin{array}{llllll}
2 & 0 & 0 & 0 & 1 & 0
\end{array}\right]^{T} \text { and } } \\
& {\left[\begin{array}{llllll}
4.2825 & -1.5159 & 0.299 & -0.1892 & 0.9776 & 0.09275
\end{array}\right]^{T} . }
\end{aligned}
$$

Using the above three solutions, a moving platform can be constructed by taking them in the order as $\left[\mathbf{b}_{i} \mathbf{j}_{i}\right]^{T}$ respectively for $i=1,2$ and 3 and the dimensions of the fixed platform corresponding to them (i.e. $\mathbf{a}_{i}$ for $i=1,2,3$ ) can be determined in the second step.

\subsection{Determination of the location of revolute joints $\mathbf{a}_{i}$ and the parameter $\left(D_{m}\right)_{i}$}

For the three solutions obtained in section $5 \cdot 1$, the corresponding positions of the revolute joints and the respective parameters $\left(D_{m}\right)_{i}$ are obtained by optimizing, using Genetic Algorithms, the Eq. (17) subject to the physical constraints: $\psi_{i k}<\psi_{\max } / 2(i=1,2,3$ and $k=1,2, \ldots, n)$ for different values of $\psi_{\max }$. Some of the results obtained for different values of $\psi_{\max }$ are shown in table 2 .

\section{Conclusion}

A two-step procedure for the dimensional synthesis of a spatial 3-RPS parallel manipulator, considering physical constraints on the motion of spherical joints, was presented. Using the procedure, it is possible to determine the dimensions of the fixed and moving platforms, direction of the revolute joints and the orientation of the spherical joints (the parameters $\left.\left(D_{m}\right)_{i}\right)$ in order that a point on the moving platform passes through a prescribed number of positions in space. In this procedure, there is no limitation on the number of positions that can be prescribed, through which a point on the moving platform can pass. As the orientation of the spherical joints influences the range of motion of the moving platform, it is absolutely necessary to consider the orientation of the spherical joint as one of the synthesis parameters in order to have more flexibility in the design of the manipulator rather than taking a fixed value for the parameter $D_{m}$. 
Table 2. Locations of the revolute joints and the values of the parameter $D_{m}$.

\begin{tabular}{|c|c|c|c|c|}
\hline & $\psi_{\max }=45^{\circ}$ & $\psi_{\max }=60^{\circ}$ & $\psi_{\max }=80^{\circ}$ & $\psi_{\max }=100^{\circ}$ \\
\hline$\left\lceil a_{1 x}\right\rceil$ & {$[3 \cdot 5227]$} & {$[2 \cdot 9884]$} & {$[2.5263]$} & {$[3.9665]$} \\
\hline$a_{1 y}$ & 1.6573 & 1.133 & $1 \cdot 1456$ & 1.054 \\
\hline$\left[a_{1 z}\right]$ & $3 \cdot 21$ & 2.975 & $3 \cdot 01$ & $2 \cdot 485$ \\
\hline$\left(D_{m}\right)_{1}$ & $2 \cdot 5$ & $2 \cdot 1$ & 1.9 & 1.5 \\
\hline$\left\lceil a_{2 x}\right]$ & {$[3.706]$} & {$[2 \cdot 8779]$} & 4 & {$[3 \cdot 5323]$} \\
\hline$a_{2 y}$ & 0.529 & $0 \cdot 1217$ & 0.9946 & 0.2198 \\
\hline$\left\lfloor a_{2 z}\right]$ & [2.857 & {$[2 \cdot 8239]$} & $2 \cdot 39$ & $2 \cdot 0336$ \\
\hline$\left(D_{m}\right)_{2}$ & $1 \cdot 5$ & $2 \cdot 1$ & 1.7 & $1 \cdot 1$ \\
\hline$\left\lceil a_{3 x}\right\rceil$ & {$[5.9263]$} & {$[5.6546]$} & $5 \cdot 0$ & {$[4.9627]$} \\
\hline$a_{3 y}$ & -1.9011 & -1.5966 & -1.7602 & -1.4522 \\
\hline$\left[a_{3 z}\right]$ & 2.4268 & 2.4814 & $2 \cdot 5034$ & 2.5905 \\
\hline$\left(D_{m}\right)_{3}$ & $0 \cdot 9$ & $1 \cdot 3$ & 1.7 & $2 \cdot 3$ \\
\hline
\end{tabular}

\section{References}

Chen P, Roth B 1967 Design equations for finitely and infinitesimally separated position synthesis of binary link and combined link chains. ASME J. Engineering for Industry 91: 209-219

Deb K 2001 Multi-objective optimization using evolutionary algorithms John Wiley and Sons

Gough V E, Whitehall S G 1962 Universal tyre test machine. Proc. of the $9^{\text {th }}$ International Congress of Fédération International des Sociétés d'Ingénieurs des Techniques de l'Automobile 117: 117-135

Lee K, Shah D K 1987 Kinematic analysis of a three degrees of freedom in-parallel actuated manipulator. Proc. IEEE International Conference on Robotics and Automation 1: 345-350

Lee K, Shah D K 1988 Dynamic analysis of a three degrees of freedom in-parallel actuated manipulator. IEEE Tran. Rob. Autom. 4: 361-367

Rao N M, Rao K M 2006 Multi-position dimensional synthesis of a spatial 3-RPS parallel manipulator. ASME J. Mech. Des. 128: 815-819

Rao N M, Rao K M 2009 Dimensional synthesis of a spatial 3-RPS parallel manipulator for a prescribed range of motion of spherical joints. Mechanism and Machine Theory 44: 477-486

Stewart D 1965 A platform with six degrees of freedom. Proc. Inst. Mech. Eng. London 180(15): 371-386

Su H J, McCarthy J M 2003 Kinematic synthesis of RPS serial Chains. ASME Proc. of the DETC2003/DAC-48813, Chicago, Illinois, USA

Tsai L W 1999 Robot analysis: The mechanics of serial and parallel manipulator (New York: John Wiley \& Sons) 142-151

Tsai L W, Kim H S 2003 Kinematic synthesis of a spatial 3-RPS parallel manipulator. ASME J. Mech. Des. 125: 92-97 